\title{
ADIABATIC INVARIANCE AND APPLICATIONS: FROM MOLECULAR DYNAMICS TO NUMERICAL WEATHER PREDICTION
}

COLIN JOHN COTTER ${ }^{1}$ and SEBASTIAN REICH ${ }^{2}$

1 Department of Mathematics, Imperial College

180 Queen's Gate, London, SW72AZ. email: colin.cotter@ic.ac.uk

${ }^{2}$ Department of Mathematics, Imperial College

180 Queen's Gate, London, SW7 2AZ. email: s.reich@ic.ac.uk

\begin{abstract}
.
A wide class of Hamiltonian systems exhibit a mixture of slow motion with superimposed fast oscillations. Under the assumption of scale separation, these systems can be investigated using the principle of adiabatic invariance. In this paper, we start with a review of some of the main theoretical and numerical findings. We then briefly summarize a few important implications for molecular dynamics (MD) before we provide a more extensive discussion of numerical weather prediction (NWP). In particular, the conservative Hamiltonian particle-mesh (HPM) method is extended to Euler's equation and the fundamental concepts of geostrophic and hydrostatic balance are illustrated on the level of 'fluid blobs'. We also demonstrate numerically that symplectic timestepping methods are able to maintain hydrostatic balance to high accuracy.
\end{abstract}

AMS subject classification: 76M28,70-08,65P10,70K70,70H11

Key words: adiabatic invariants, symplectic integration, molecular dynamics, numerical weather prediction, highly oscillatory Hamiltonian systems.

\section{Introduction}

In this paper, we review some basic results about highly oscillatory Hamiltonian systems and adiabatic invariants. We show how these concepts can be utilized in the context of molecular dynamics (MD) and numerical weather prediction (NWP). While Hamiltonian techniques and symplectic integration methods have been extensively discussed in the context of MD (see, for example, SKEEL [32]), the application to NWP is new. Hence we will mainly focus on NWP in this paper. A necessary and fundamental step, which we outline in $§ 3.2 .2$, is the spatial discretization of Euler's equation into a conservative system of interacting particles. Once this step has been taken, well-known balance concepts, such as hydrostatic and geostrophic balance [1], can be discussed quite naturally in the context of classical mechanics and adiabatic invariance. One can expect that symplectic integration methods will faithfully respect such balance principles on a discrete level. We demonstrate this in $\S 3.2 .4$ for a simple two-dimensional hydrostatic system. On the other hand, all known grid-based 
discretization methods for Euler's equation are non-Hamiltonian and balance typically requires the introduction of numerical dissipation.

\section{Adiabatic invariants and symplectic integration methods}

In the following four subsections, we introduce the concept of adiabatic invariance in the context of several increasingly complex model systems.

\subsection{Slowly varying harmonic oscillator}

The harmonic oscillator with slowly changing frequency $\omega$ is the simplest example of a fast-slow Hamiltonian system, i.e.

$$
y^{\prime \prime}=-\omega(\varepsilon \tau)^{2} y,
$$

where $\varepsilon>0$ is a small parameter measuring the separation of scales between the fast oscillations in $y$ and the slowly-varying frequency $\omega=\mathcal{O}\left(\varepsilon^{0}\right)$. Upon a rescaling of time $\tau$ to $t=\varepsilon \tau$, we can write the equation of motion in the following form which is more convenient for later use:

$$
\ddot{y}=-\varepsilon^{-2} \omega(t)^{2} y .
$$

As easily checked, this system does not conserve energy. However, consider the action

$$
J=\frac{1}{\omega(t)}\left(\dot{y}^{2}+\varepsilon^{-2} \omega(t)^{2} y^{2}\right)
$$

and its time derivative

$$
\dot{J}=\dot{\omega}\left(\varepsilon^{-2} y^{2}-\omega^{-2} \dot{y}^{2}\right) .
$$

Over one (short) period $T=2 \varepsilon \pi / \omega(t)$, the motion in $y(t)$ is approximately harmonic with frequency $\omega / \varepsilon$ and we find that

$$
J(t+T)-J(t)=\int_{t}^{t+T} \dot{J}(s) d s \approx \dot{\omega}(t) \int_{t}^{t+T}\left(\varepsilon^{-2} y(s)^{2}-\omega(t)^{-2} \dot{y}(s)^{2}\right) d s \approx 0 .
$$

A more careful calculation, taking into account slow changes in $\omega$ and $\dot{\omega}$, yields the estimate

$$
\frac{J(t+T)-J(t)}{J(t)}=\mathcal{O}\left(\varepsilon^{2}\right) .
$$

From this result we can conclude that

$$
\frac{|J(t)-J(0)|}{|J(0)|}=\mathcal{O}(\varepsilon) \quad \text { for } \quad|t|=\mathcal{O}\left(\varepsilon^{0}\right) .
$$

Using sophisticated normal form theory, Neishtadt [22] was able to dramatically improve this result under the assumption of analytic $\omega(t)$ to the following estimate:

$$
\frac{|J(0)-J(t)|}{|J(0)|}=\mathcal{O}(\varepsilon) \quad \text { for } \quad|t|=\mathcal{O}\left(\mathrm{e}^{c / \varepsilon}\right)
$$


The differential equation (2.1) can be discretized by the Störmer-Verlet method [15] once the conjugate momentum $p_{y}=\dot{y}$ is introduced. The resulting one-step map is symplectic and we conclude from backward error analysis $[3,14,25]$ that there is a modified time-dependent Hamiltonian

$$
\tilde{H}=\frac{1}{2}\left(p_{y}^{2}+\varepsilon^{-2} \omega(t)^{2} y^{2}\right)+(\Delta t / \varepsilon)^{2} \Delta H\left(y, p_{y}, t ; \Delta t / \varepsilon\right)
$$

such that its time- $\Delta t$-flow is exponentially close to the Störmer-Verlet map for $\Delta t / \varepsilon \ll 1$. Furthermore, one can show that $J$ is also an adiabatic invariant for the modified Hamiltonian $\tilde{H}$ and, provided that

$$
\Delta t=\mathcal{O}\left(\varepsilon^{2}\right),
$$

the estimate (2.2) also holds for the numerical method. See also the paper by Shimada \& Yoshida [31]. Condition (2.3) represents a severe step-size restriction which is not desirable for long time simulations as linear stability alone merely dictates that $\Delta t=\mathcal{O}(\varepsilon)$. In fact, a step-size $\Delta t=\mathcal{O}(\varepsilon)$ often gives excellent results.

\subsection{A simple slow-fast Hamiltonian system}

In this and the subsequent section we demonstrate how the simple example of a slowly varying harmonic oscillator carries over to more complex Hamiltonian systems. We start by considering a two degrees-of-freedom (DoF) Hamiltonian system of the form

$$
\begin{aligned}
\ddot{x} & =-\frac{y^{2}}{2 \varepsilon^{2}} \nabla_{x} K(x)-\nabla_{x} V(x, y), \\
\ddot{y} & =-\frac{1}{\varepsilon^{2}} K(x) y-\nabla_{y} V(x, y),
\end{aligned}
$$

where $\varepsilon>0$ is a small parameter and $K(x)=\mathcal{O}\left(\varepsilon^{0}\right)$. The system is canonical Hamiltonian with energy

$$
\mathcal{E}=\frac{1}{2}\left[\dot{x}^{2}+\dot{y}^{2}+\varepsilon^{-2} K(x) y^{2}\right]+V(x, y) .
$$

The first observation is that $y(t)=\mathcal{O}(\varepsilon)$ for dynamics with bounded total energy. Furthermore, it is intuitively clear that the motion in $x(t)$ is slow while $y(t)$ is highly-oscillatory with an approximate equilibrium value $y_{o}=0$. The fast oscillations in $y$ are essentially described by the harmonic oscillator

$$
\ddot{y} \approx-\frac{1}{\varepsilon^{2}} K(x(t)) y
$$

with frequency $\omega=\sqrt{K(x(t))} / \varepsilon$ depending on $x(t)$. Hence we are back to the situation described in the previous subsection. More precisely, the result by NEISHTADT [22] suggests that the associated adiabatic invariant

$$
J(t)=\frac{1}{\sqrt{K(x(t))}}\left(\dot{y}(t)^{2}+\varepsilon^{-2} K(x(t)) y(t)^{2}\right)
$$


is preserved over time intervals of $\mathcal{O}\left(e^{c / \varepsilon}\right)$ up to terms of $\mathcal{O}(\varepsilon)$. This statement has been made precise in REICH [28]. See the Appendix for a short outline of the basic ideas. An important consequence of this result is that the slow DoF approximately moves along the constant energy surface

$$
\mathcal{E}_{\text {red }}=\frac{1}{2} \dot{x}^{2}+V(x, 0)+\frac{J(0)}{2} \sqrt{K(x)} .
$$

The associated reduced canonical equations of motion in the variable $x$ are

$$
\ddot{x}=-\nabla_{x} V(x, 0)-\frac{J(0)}{2} \nabla_{x} \sqrt{K(x)} .
$$

These equations were first derived by RuBin \& UnGAR [29].

Again it is relatively easy to show that a symplectic integrator will capture the correct slow dynamics provided the step-size $\Delta t$ satisfies $\Delta t \sim \varepsilon^{2}$. In particular, the reduced energy (2.7) will be approximately preserved over exponentially long times for analytic $K(x)$ and $V(x, y)$. See ReIch [26] for details. Note that the same statement is not true for general numerical methods.

\subsection{More general slow-fast Hamiltonian systems}

We have seen in the previous subsection that the principle of adiabatic invariance is a powerful tool to eliminate a single fast DoF in Hamiltonian systems. One would like to extend this result to slow-fast Hamiltonian systems with many DoF, e.g., consider

$$
\begin{aligned}
\ddot{\mathbf{x}} & =-\frac{1}{2 \varepsilon^{2}} \nabla_{\mathbf{x}}\left[\mathbf{y}^{T} \mathbf{K}(\mathbf{x}) \mathbf{y}\right]-\nabla_{\mathbf{x}} V(\mathbf{x}, \mathbf{y}), \\
\ddot{\mathbf{y}} & =-\frac{1}{\varepsilon^{2}} \mathbf{K}(\mathbf{x}) \mathbf{y}-\nabla_{\mathbf{y}} V(\mathbf{x}, \mathbf{y}),
\end{aligned}
$$

where $\mathbf{x} \in R^{n}, \mathbf{y} \in R^{m}$, and $\mathbf{K}(\mathbf{x})$ is a symmetric positive-definite matrix-valued function of $\mathbf{x}$.

Solutions oscillate about an approximate equilibrium point $\mathbf{y}_{o}=\mathbf{0}$. The fast oscillations in $\mathbf{y}$ are characterized by the linear system

$$
\ddot{\mathbf{y}}=-\varepsilon^{-2} \mathbf{K}(t) \mathbf{y},
$$

$\mathbf{K}(t)=\mathbf{K}(\mathbf{x}(t))$. One can associate an action variable $J_{i}$ with every (timevarying) eigenvalue $\lambda_{i}(t)$ of the symmetric matrix $\mathbf{K}(t)$. The associated normal mode has frequency $\omega_{i}=\sqrt{\lambda_{i}}$. The action variables can undergo rapid transitions of order one near one-to-one resonances which occur whenever $\omega_{i}\left(t^{\prime}\right) \approx \omega_{j}\left(t^{\prime}\right)$ for some time $t^{\prime}$ and indices $i \neq j$. Hence a closed form reduction, as found for single fast DoF systems, is no longer feasible in general. See Takens [33] and Bornemann [5] for a rigorous treatment. On the other hand, numerical experiments indicate (see, e.g., REICH [27] and the numerical example below) that the total adiabatic invariant $J=\sum_{i=1}^{m} J_{i}$ drifts very little and essentially behaves like an adiabatic invariant over relatively long periods of time. This implies that solutions with a small initial value of $J$ will stay close to $\mathbf{y}_{o}$ for long periods of time. 


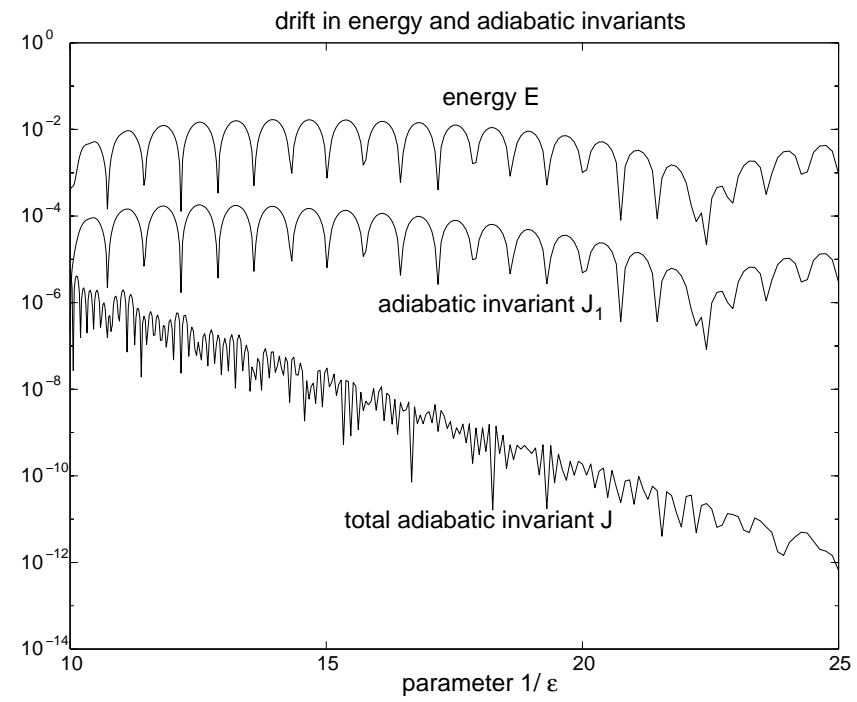

Figure 2.1: The plot shows the difference $\left|E\left(t_{1}\right)-E\left(t_{0}\right)\right|$ in energy, the difference $\left|J\left(t_{1}\right)-J\left(t_{0}\right)\right|$ in the total adiabatic invariant, and the difference $\left|J_{1}\left(t_{1}\right)-J_{1}\left(t_{0}\right)\right|$ in the adiabatic invariant $J_{1}$ as a function of $\varepsilon$.

\subsection{Rapid oscillations about a slow manifold}

We have assumed in the previous two subsections that slow and fast variables had already been identified. Such a setting is not normally given for problems from physics or other applications. In fact, part of the challenge of slow-fast dynamics is to find a proper change of variables that leads from the original equations to equations of the form (2.9)-(2.10), at least locally. In terms of the original variables, the equation $\mathbf{y}=\mathbf{y}_{o}$ corresponds to a manifold (often called a 'slow' manifold) and $\mathbf{Y}=\mathbf{y}-\mathbf{y}_{o}$ contains fast oscillations normal to that manifold. See the recent survey by MACKAY [21] for a careful discussion of the concept of 'slow' manifolds.

\subsection{A numerical experiment}

We demonstrate the drift in action variables by means of a simple toy problem. For our purposes it is sufficient to consider a time-dependent system with two fast DoF:

$$
\begin{aligned}
& \ddot{y}_{1}=-\frac{\omega_{1}(t)^{2}}{\varepsilon^{2}} y_{1}-e^{-t^{2} / 2}\left[\left(y_{2}-y_{1}\right)+\left(y_{2}-y_{1}\right)^{3}\right], \\
& \ddot{y}_{2}=-\frac{\omega_{2}(t)^{2}}{\varepsilon^{2}} y_{2}-e^{-t^{2} / 2}\left[\left(y_{1}-y_{2}\right)+\left(y_{1}-y_{2}\right)^{3}\right],
\end{aligned}
$$

with frequencies

$$
\omega_{i}(t)=\sqrt{1+(-1)^{i}\left(0.5-\tanh t^{2}\right)} .
$$


The two associated action variables are

$$
J_{i}=\frac{1}{\omega_{i}}\left(\dot{y}_{i}^{2}+\frac{\omega_{i}^{2}}{\varepsilon^{2}} y_{i}^{2}\right)
$$

and $J=J_{1}+J_{2}$. The energy of the system is given by

$$
E=\frac{1}{2} \sum_{i=1,2}\left(\dot{y}_{i}^{2}+\frac{\omega_{i}(t)^{2}}{\varepsilon^{2}} y_{i}^{2}\right)-\frac{1}{4} e^{-t^{2} / 2}\left[2\left(y_{2}-y_{1}\right)^{2}+\left(y_{1}-y_{2}\right)^{4}\right]
$$

Note that time $t$ takes the role of the slow variable $x$. We start the simulation with initial conditions $y_{1}\left(t_{0}\right)=y_{2}\left(t_{0}\right)=0, \dot{y}_{1}\left(t_{0}\right)=\dot{y}_{2}\left(t_{0}\right)=1$ and $t_{0}=-10$. The simulation is stopped at $t_{1}=10$. The parameter $\varepsilon$ is taken from the interval $[0.04,0.1]$. Note that at both $t_{0}$ and $t_{1}$ the time-dependence of the system has effectively vanished and that the two oscillators essentially decouple. This property is the reason behind the special choice of the energy $E$ and the frequencies $\omega_{i}$.

We observe from Fig. 2.1 the following qualitative behavior for the total adiabatic invariant $J$ :

$$
\left|J\left(t_{1}\right)-J\left(t_{0}\right)\right|=\mathcal{O}\left(\mathrm{e}^{-c / \varepsilon}\right) .
$$

The exponential dependence in (2.11) is remarkable and is currently not supported by rigorous mathematical results. The same exponential dependence was also found for other choices of the coupling term and the fast frequencies.

\section{Applications}

In the following two sections, we discuss applications of adiabatic invariance to molecular dynamics (MD) and numerical weather prediction (NWP). After a short survey of adiabatic invariance in the context of $\mathrm{MD}$, the main focus will be on NWP.

\subsection{Molecular dynamics (MD)}

Bond stretching and bending modes are responsible for the fastest oscillations in molecular systems. However a clear scale separation is often not given and the concept of adiabatic invariance is only applicable in special circumstances such as hydrogen bonds. Nevertheless the concepts of averaging and 'slow' manifolds have found important applications in MD. We start with the concept of a 'slow' manifold. Molecular systems often oscillate about some mean configuration and one can, under certain circumstances, ignore those oscillations and (partially) restrict the molecule to its mean configuration. Mathematically, the mean configuration describes a submanifold in configuration space, called the 'slow' manifold. For example, one can set bond stretching modes equal to their equilibrium value, i.e. $\left\|\mathbf{r}_{i}-\mathbf{r}_{j}\right\|=l_{0}$. This equation defines a holonomic constraint [2], which can be enforced numerically using SHAKE or RATTLE. These two numerical methods were shown to be symplectic by LEIMKUHLER \& SKEEL 
[19]. A better approximation to the 'slow' manifold can be achieved by minimizing the potential (or total) energy along the bond stretching modes. The significance of this improvement to transition rates has been demonstrated by REICH [24]. The idea of elastic constraints has been implemented numerically by Zhou, Reich \& Brooks [34]. See also Hess, Saint-Martin \& Berendsen $[16]$.

The true motion of a molecular system will oscillate about the 'slow' manifold as defined by the equilibrium values of bond stretching and bending modes. These fast oscillations can, in principle, be discussed using the concept of adiabatic invariance and averaging. Local coordinate transformations, which lead from the MD equations of motion to equations similar to those of $\S 2.3$, can be found in Takens [33], Bornemann [5] and Reich [23, 27]. However, resonances between fast modes make an explicit elimination of these DoF impossible within the context of classical mechanics. A successful application of averaging has recently been proposed by Garcia-Archilla, Sanz-Serna \& Skeel [12]. They suggested to build local-in-time averaging into symplectic integration methods and to thus allow for large time-steps $\Delta t=\mathcal{O}\left(\varepsilon^{0}\right)$. Such large time-step (LTS) methods have been successfully implemented for MD by IzaguirRe, REICH \& Skeel [17]. See the monograph [15] for related work on LTS methods by Hairer, Hochbruck \& Lubich and also Leimkuhler \& Reich [18].

\subsection{Numerical Weather Prediction (NWP)}

We come now to the main motivation for this paper; namely numerical weather prediction (NWP). For an introduction to atmospheric dynamics see [1]. Numerical aspects are discussed, for example, in DURRAN [8].

We propose an extension of the Hamiltonian particle-mesh (HPM) method of Frank, GotTwald \& Reich [9] to the compressible three-dimensional Euler equations. This conservative truncation allows us to discuss geostrophic and hydrostatic balance in the context of adiabatic invariance. Following general results outlined earlier, we anticipate that symplectic methods will preserve balance to high accuracy and over long times. Furthermore, the HPM method conserves the Rossby-Ertel potential vorticity [1]. This can be shown along the lines of Frank \& Reich [10] and Bridges, Hydon \& Reich [6].

The only other two publications on particle methods for NWP we are aware of are SALmon [30] and GADIAN [11]. Both references use the SPH method of LuCy [20] and Monaghan \& Gingold [13] and apply it to two-dimensional (vertical and horizontal) atmospheric model systems. 


\subsubsection{Euler equations of atmospheric motion}

The Euler equations for inviscid isentropic motion of a perfect gas in a rotating reference frame can be expressed in the form [8]:

$$
\begin{aligned}
\frac{d \mathbf{v}}{d t} & =-\theta \nabla_{\mathbf{x}} \pi-g \mathbf{k}-f \mathbf{k} \times \mathbf{v}, \\
\frac{d \theta}{d t} & =0 \\
\frac{d \pi}{d t} & =-\frac{R}{c_{v}} \pi \nabla_{\mathbf{x}} \cdot \mathbf{v},
\end{aligned}
$$

where pressure $p$, as used in the standard formulation of Euler's equations, has been replaced by Exner's function

$$
\pi=c_{p}\left(p / p_{0}\right)^{R / c_{p}}=c_{p} \frac{T}{\theta}
$$

which gives rise to the relation $\rho^{-1} \nabla_{\mathbf{x}} p=\theta \nabla_{\mathbf{x}} \pi$.

In the preceding, $g$ is the gravitational acceleration, $f$ is twice the angular velocity of the frame of reference, ${ }^{1} \mathbf{k}=(0,0,1)^{T}$ is a unit vector pointing in the $z$-direction for simplicity, $d() / d t=\partial_{t}()+\mathbf{v} \cdot \nabla_{\mathbf{x}}()$ is the material time derivative, $\mathbf{v}=(u, v, w)^{T}$ is the three-dimensional velocity vector, $\theta=T\left(p / p_{0}\right)^{-R / c_{p}}$ is the potential temperature, $T$ is the temperature, $\rho$ is the density, $p_{0}$ is a constant reference pressure, $R$ is the gas constant for dry air, $c_{p}$ is the specific heat at constant pressure, $c_{v}$ is the specific heat at constant volume, $c_{p}=R+c_{v}=$ $1005 \mathrm{~J} \mathrm{~K}^{-1} \mathrm{~kg}^{-1}$, and $R / c_{v} \approx 0.4, c_{p} / c_{v} \approx 1.4$.

Exner's function can also be expressed in the form [8]

$$
\pi=c_{p}\left(\frac{\rho \theta}{\rho_{0} T_{0}}\right)^{R / c_{v}}, \quad \rho_{0} T_{0}=p_{0} / R .
$$

This suggests to introduce a new density $\mu=\rho \theta /\left(\rho_{0} T_{0}\right)$ and to replace the equations (3.1)-(3.3) by the modified set

$$
\begin{aligned}
& \frac{d \mathbf{v}}{d t}=-\theta \nabla_{\mathbf{x}} \pi-g \mathbf{k}-f \mathbf{k} \times \mathbf{v}, \\
& \frac{d \theta}{d t}=0, \\
& \frac{d \mu}{d t}=-\mu \nabla_{\mathbf{x}} \cdot \mathbf{v},
\end{aligned}
$$

with Exner's function now taking the form $\pi=c_{p} \mu^{R / c_{v}}$.

External heating or cooling of the atmosphere can be taken into account by replacing equation (3.5) by

$$
\frac{d \theta}{d t}=\frac{Q \theta}{c_{p} T}=\frac{Q}{\pi}
$$

\footnotetext{
${ }^{1}$ The angular velocity is assumed, for simplicity, to be constant. This is often referred to as the $f$-plane approximation. A more realistic approximation is provided by the $\beta$-plane approximation $f=f_{0}+\beta y$.
} 
where $Q$ is the diabatic heating rate per unit mass.

\subsubsection{Hamiltonian truncation of Euler's equations}

The equations (3.4)-(3.6) can be discretized by a natural extension of the Hamiltonian particle-mesh (HPM) method. In particular, given a computational grid $\left\{\mathbf{x}_{i}\right\}$ and $K$ Lagrangian particles $\left\{\mathbf{X}_{k}(t)\right\}$, an approximation to $\mu$ at the grid point $\mathbf{x}_{i}$ is provided by

$$
\mu_{i}(t)=\frac{1}{\gamma_{i} \rho_{0} T_{0}} \sum_{k} m_{k} \theta_{k} \psi_{i}\left(\mathbf{X}_{k}(t)\right) .
$$

Here $\psi_{i}(\mathbf{x})$ are positive basis functions, which form a partition of unity $\sum_{i} \psi_{i}(\mathbf{x})=1$, the constants $\gamma_{i}$ are defined by $\gamma_{i}=\int \psi_{i}(\mathbf{x}) d \mathbf{x}, m_{k}$ is the mass of the $k$-th particle and $\theta_{k}$ its potential temperature. An important identity is the interpolation formula

$$
\pi(\mathbf{x}, t)=\sum_{i} \psi_{i}(\mathbf{x}) \pi_{i}(t)
$$

which implies, for example,

$$
\nabla_{\mathbf{x}} \pi(\mathbf{x}, t)=\sum_{i} \pi_{i}(t) \nabla_{\mathbf{x}} \psi_{i}(\mathbf{x})
$$

Next we define $e_{i}=e\left(\mu_{i}\right)$ such that $e^{\prime}\left(\mu_{i}\right)=\rho_{0} T_{0} \pi_{i}$ and $\pi_{i}=c_{p}\left[\mu_{i}(t)\right]^{R / c_{v}}$. A simple calculation yields

$$
\nabla \mathbf{X}_{k}\left(\sum_{i} \gamma_{i} e_{i}(t)\right)=m_{k} \theta_{k} \sum_{i} \pi_{i} \nabla_{\mathbf{X}_{k}} \psi_{i}\left(\mathbf{X}_{k}\right)=m_{k} \theta_{k} \nabla_{\mathbf{x}} \pi(\mathbf{x}, t)_{\mid \mathbf{x}=\mathbf{X}_{k}}
$$

Here the gradient w.r.t. $\mathbf{X}_{k}=\left(X_{k}, Y_{k}, Z_{k}\right)^{T}$ stands for the column vector $\nabla_{\mathbf{X}_{k}}()=\left(\partial_{X_{k}}(), \partial_{Y_{k}}(), \partial_{Z_{k}}()\right)^{T}$.

Using these identities, the momentum equation (3.4) is now discretized to

$$
\frac{d}{d t} \mathbf{v}_{k}=-\theta_{k} \sum_{i} \pi_{i} \nabla_{\mathbf{X}_{k}} \psi_{i}\left(\mathbf{X}_{k}\right)-g \mathbf{k}-f \mathbf{k} \times \mathbf{v}_{k},
$$

and particles are advected according to

$$
\frac{d}{d t} \mathbf{X}_{k}=\mathbf{v}_{k}
$$

for $k=1, \ldots, K$. The discrete equations of motion are Hamiltonian with conserved energy

$$
\mathcal{E}=\frac{1}{2} \sum_{k} m_{k}\left\|\mathbf{v}_{k}\right\|^{2}+g \sum_{k} m_{k} \mathbf{k} \cdot \mathbf{X}_{k}+\sum_{i} \gamma_{i} e\left(\mu_{i}\right) .
$$


The (non-canonical) symplectic two-form is given by

$$
\omega=\sum_{k} m_{k} d \mathbf{v}_{k} \wedge d \mathbf{X}_{k}+\frac{f}{2} \sum_{k} m_{k} d \mathbf{X}_{k} \wedge\left(\mathbf{k} \times d \mathbf{X}_{k}\right)
$$

Smoothing, as used in the original HPM method, can be achieved by introducing the smoothed/filtered density $\tilde{\mu}=\left(1-\alpha^{2} \nabla^{2}\right)^{-1} \mu$ and its corresponding numerical approximation

$$
\tilde{\mu}_{i}=a_{i j} \mu_{j}
$$

where $\left\{a_{i j}\right\}$ is a discrete approximation to the inverse modified Helmholtz operator. The smoothing operator essentially filters out fast sound waves with a wave length shorter than the smoothing length. This allows for larger time-steps with an explicit symplectic method such as Störmer-Verlet.

To obtain a closed system of equations, appropriate boundary conditions need to be imposed. However, we do not need to specify them for the purpose of this paper and will not dwell on this issue here any further.

\subsubsection{Geostrophic and hydrostatic balance}

Large scale atmospheric flow regimes are characterized by the relative smallness of the acceleration term on the left hand side of (3.1) compared to the forcing terms on the right hand side of the equation. Consider, for example, the vertical momentum equation. Following [1], the vertical acceleration can be estimated to

$$
\frac{d}{d t} w \sim 10^{-7} \mathrm{~ms}^{-2}
$$

which is much smaller than $g \sim 10 \mathrm{~m} \mathrm{~s}^{-2}$. The only remaining term is $\theta \partial \pi / \partial z$, which then has to be in balance with the gravitational forcing term. More generally, we are led to the numerical approximation

$$
\mathbf{0} \approx-\theta_{k} \sum_{i} \pi_{i} \nabla_{\mathbf{X}_{k}} \psi_{i}\left(\mathbf{X}_{k}\right)-g \mathbf{k}-f \mathbf{k} \times \mathbf{v}_{k}
$$

Exact equality in (3.12) defines a manifold $\mathcal{S}$ of dimension $d=3 K$ in the space of all particle locations and velocities. The first two components in equation (3.12) yield the (numerical) geostrophic balance conditions

$$
\begin{aligned}
& f v_{k}=+\theta_{k} \sum_{i} \pi_{i} \partial_{X_{k}} \psi_{i}\left(\mathbf{X}_{k}\right) \\
& f u_{k}=-\theta_{k} \sum_{i} \pi_{i} \partial_{Y_{k}} \psi_{i}\left(\mathbf{X}_{k}\right)
\end{aligned}
$$

while the last component gives rise to the (numerical) hydrostatic balance relation

$$
0=-\theta_{k} \sum_{i} \pi_{i} \partial_{Z_{k}} \psi_{i}\left(\mathbf{X}_{k}\right)-g
$$


which acts as a holonomic constraint on the particle locations. Upon differentiating this constraint with respect to time and making use of the geostrophic balance relations (3.13)-(3.14), one obtains an algebraic equation for the vertical particle velocity $w_{k}$. This hidden velocity constraint, equation (3.15), and equations (3.13)-(3.14) define the proper 'slow' manifold $\mathcal{S}_{p}$ of dimension $2 K$.

Note that setting the acceleration equal to zero would corresponds in molecular dynamics to finding a static minimum of the potential energy landscape. On the other hand, equation (3.12) involves the horizontal particle velocities and leads to (reduced) dynamic equations of motion on the 'slow' manifold $\mathcal{S}_{p}$. These reduced equations are, in fact, constrained Euler-Lagrange equations with (degenerate) Lagrangian

$$
\mathcal{L}=\frac{f}{2} \sum_{k} m_{k} \mathbf{X}_{k} \cdot\left(\mathbf{k} \times \dot{\mathbf{X}}_{k}\right)+g \sum_{k} m_{k} \mathbf{k} \cdot \mathbf{X}_{k}+\sum_{i} \gamma_{i} e\left(\mu_{i}\right) .
$$

We now go back to the full equations (3.9)-(3.10) and investigate the solution behaviour near the 'slow' manifold $\mathcal{S}_{p}$. Let us consider hydrostatic balance first. Assume we are given a set of static particle locations $\mathbf{X}_{k}^{0}$, which are in perfect balance, i.e.

$$
0=\theta_{k} \sum_{i} \pi\left(\mu_{i}^{0}\right) \partial_{Z_{k}} \psi_{i}\left(\mathbf{X}_{k}^{0}\right)+g, \quad \mu_{i}^{0}=\frac{1}{\gamma_{i} \rho_{0} T_{0}} \sum_{k} m_{k} \theta_{k} \psi_{i}\left(\mathbf{X}_{k}^{0}\right)
$$

Next we slightly perturb the location of one of the particles in the $z$-direction. Then, following the standard linearization arguments, such a perturbation $\delta Z_{k}$ approximately satisfies the linear second-order differential equation

$$
\delta \ddot{Z}_{k} \approx-\theta_{k} \sum_{i} \pi\left(\mu_{i}^{0}\right) \partial_{Z_{k}} \psi_{i}\left(\mathbf{X}_{k}^{0}+\delta Z_{k} \mathbf{k}\right)+g \approx-\theta_{k} \sum_{i} \pi\left(\mu_{i}^{0}\right) \partial_{Z_{k}}^{2} \psi_{i}\left(\mathbf{X}_{k}^{0}\right) \delta Z_{k},
$$

and the motion is oscillatory with frequency $\omega_{k}$ provided that

$$
\omega_{k}^{2}=\theta_{k} \sum_{i} \pi\left(\mu_{i}^{0}\right) \partial_{Z_{k}}^{2} \psi_{i}\left(\mathbf{X}_{k}^{0}\right)
$$

is positive. We also note that the hydrostatic balance condition is equivalent to

$$
\sum_{i} \pi\left(\mu_{i}^{0}\right) \partial_{Z} \psi_{i}(\mathbf{X})=-\frac{g}{\theta(\mathbf{X})}
$$

for $\mathbf{X}=\mathbf{X}_{k}^{0}$. But, in fact, one can use this relation for all $\mathbf{X}$ and, hence,

$$
\omega^{2}=-\theta \partial_{Z} \frac{g}{\theta}=\frac{g}{\theta} \frac{\partial \theta}{\partial Z},
$$

which coincides with the standard definition of the buoyancy (or Brunt-Väisälä) frequency [1]; for the lower atmosphere the corresponding period $2 \pi / \omega$ is on the order of a few minutes. 
Slow external heating or cooling, which leads to time-dependent potential temperature coefficients $\theta_{k}(t)$ via the differential equation (3.7), implies that the buoyancy frequencies $\omega_{k}$ become (slowly) time-dependent. Hence the ratio of oscillatory energy

$$
E_{k}^{\mathrm{os}}(t)=\frac{1}{2}\left[\left(\delta \dot{Z}_{k}\right)^{2}+\omega_{k}(t)^{2}\left(\delta Z_{k}\right)^{2}\right]
$$

to frequency $\omega_{k}(t)$ is a candidate for adiabatic invariance under external heating or cooling. Similar to MD simulations, this simple picture is again made more complex due to possible resonant interactions between different particles with (resonant) frequencies $\omega_{k}$ and $\omega_{l}$.

Let us now turn to geostrophic balance. The horizontal momentum equations are

$$
\begin{aligned}
& \frac{d}{d t} u_{k}=+f v_{k}-\theta_{k} \sum_{i} \pi_{i} \partial_{X_{k}} \psi_{i}\left(\mathbf{X}_{k}\right), \\
& \frac{d}{d t} v_{k}=-f u_{k}-\theta_{k} \sum_{i} \pi_{i} \partial_{Y_{k}} \psi_{i}\left(\mathbf{X}_{k}\right),
\end{aligned}
$$

with the vertical velocity $w_{k}=0$, for simplicity. We also assume that $f$ is much larger than one. Then the motion in the horizontal velocity field $\mathbf{u}=(u, v)$ is highly oscillatory about the geostrophic equilibrium values $\left(u_{k}^{g}, v_{k}^{g}\right)$ defined by equations (3.13)-(3.14), i.e.

$$
\frac{d}{d t} u_{k}^{\mathrm{ag}} \approx+f v_{k}^{\mathrm{ag}}, \quad \frac{d}{d t} v_{k}^{\mathrm{ag}} \approx-f u_{k}^{\mathrm{ag}},
$$

with the ageostrophic velocities defined by

$$
u_{k}^{\mathrm{ag}}=u_{k}-u_{k}^{\mathrm{g}}, \quad v_{k}^{\mathrm{ag}}=v_{k}-v_{k}^{\mathrm{g}} .
$$

Since $f$ is here taken to be identical for all particles, we have to expect strongly resonant interactions and only the total ageostrophic kinetic energy

$$
E_{\mathrm{ag}}(t)=\frac{1}{2} \sum_{k} m_{k}\left[\left(u_{k}^{\mathrm{ag}}\right)^{2}+\left(v_{k}^{\mathrm{ag}}\right)^{2}\right]
$$

is a candidate for adiabatic invariance. Results by Benettin, Galgani \& GIORGILLI [4] suggest that this quantity is, in fact, approximately preserved over time-periods of order $\mathcal{O}\left(e^{c f}\right), c>0$ some constant, in the formal limit $f \rightarrow \infty$ provided the Exner function $\pi$ stays sufficiently smooth. Indeed, as demonstrated for the two-dimensional shallow-water equations by FRANK, GoTTWALD \& REICH [9], smoothing (as defined by (3.11)) is essential for maintaining geostrophic balance in a particle method. See also CotTer [7]. We very much expect that the same is true for hydrostatic balance and we demonstrate this by means of the following simple test example. 


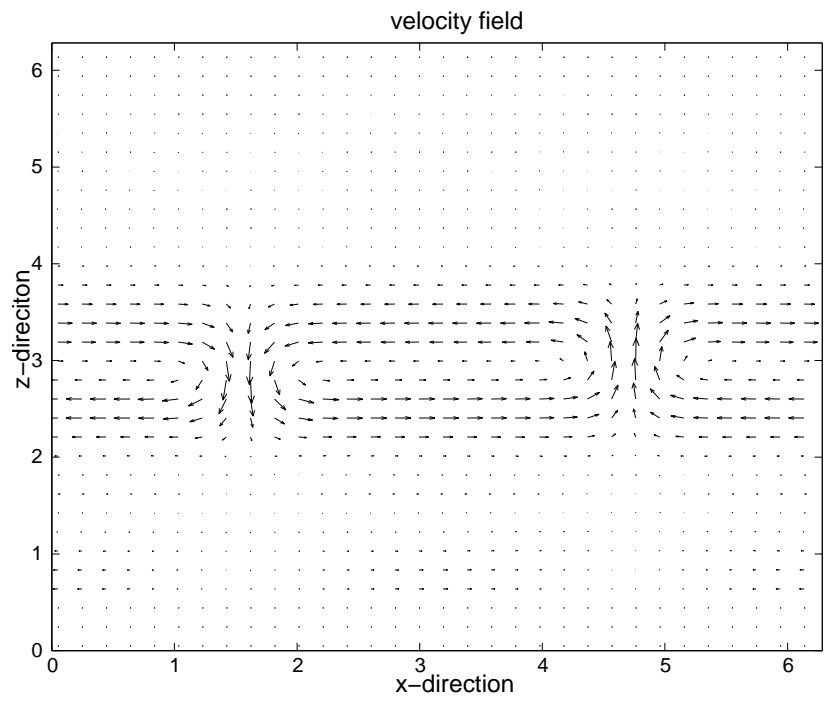

Figure 3.1: Velocity field for 'conveyor belt' circulation at time $t=15$ plotted at grid points.

\subsubsection{Numerical experiment}

Let us start by considering strictly vertical motion along the $z$-axis based upon the equations of motion

$$
\ddot{Z}=-\theta \partial_{Z} \pi-g
$$

We introduce an Eulerian grid $z_{i}=i \Delta z, i=1,2, \ldots, I$, and particles $X_{k}(t)$ such that $Z_{k}(0)>1 / 2$ for all $k=1, \ldots, K$. In fact we simply set

$$
Z_{k}(0)=\frac{\Delta z}{4} k+1 / 2
$$

Next we postulate an Exner function with grid values $\pi_{i}^{0}=10+1 /\left(z_{i}\right)^{2}$. Hence $\pi^{0}(z)=\sum_{i} \psi_{i}(z) \pi_{i}^{0}$ and hydrostatic balance dictates that

$$
\theta_{k} \partial_{Z_{k}} \pi^{0}\left(Z_{k}\right)=-g
$$

which defines the particle's potential temperature $\theta_{k}$. We finally assign to each particle a mass $m_{k}$ such that $\pi\left(\mu_{i}(t)\right) \approx \pi_{i}^{0}$ and set $R=p_{0}=R / c_{v}=c_{p}=1$, for simplicity. The discrete equations of motion are now set equal to

$$
\ddot{Z}_{k}=-\theta_{k} \sum_{i} \partial_{Z_{k}} \psi_{i}\left(Z_{k}\right)\left[\pi_{i}(t)+\pi_{i}^{0}-\pi_{i}(0)\right]-g, \quad \pi_{i}(t)=\pi\left(\mu_{i}(t)\right) .
$$

The reason for this particular setup is that the discrete system is in perfect hydrostatic balance at time $t=0$.

It is straightforward to extend this approach to a two-dimensional vertical slice model with horizontal variable denoted by $x$. We assume that initially the system 


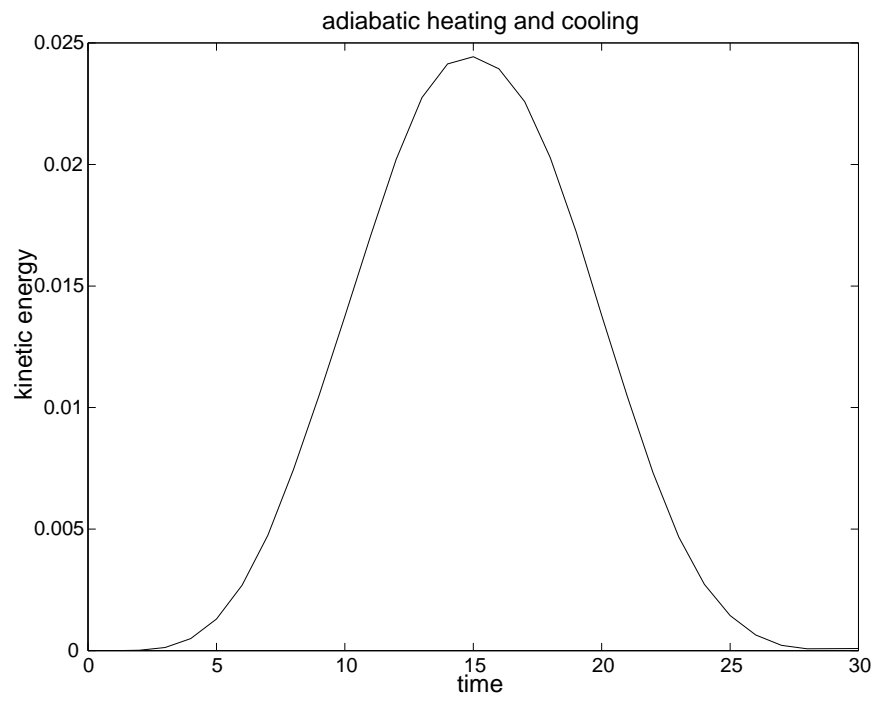

Figure 3.2: Kinetic energy relaxation after smooth (adiabatic) activation/deactivation of 'conveyor belt'.

is homogeneous in $x$ and apply periodic boundary conditions in the $x$-direction. The computational domain is taken to be equal to $(x, z) \in[0,2 \pi]^{2}$. We use a $128 \times 128$ grid, tensor product cubic B-splines as basis functions, and $K=262144$ particles. The time-step is set to $\Delta t=0.01$. The density $\mu$ is smoothed using a modified Helmholtz operator with smoothing length $\alpha=0.3927$.

Starting from zero initial velocities, an internal circulation (conveyor belt) is created by heating the system near $(X, Z)=(3 \pi / 2, \pi)$ and cooling it at $(X, Z)=$ $(\pi / 2, \pi)$. A fully activated circulation pattern at time $t=15$ is displayed in Fig. 3.1. Furthermore, the 'conveyor belt' is smoothly activated and deactivated over a time period of thirty time units. As shown in Fig. 3.2, the kinetic energy approaches zero at $t=30$ indicating that the system has returned adiabatically to a stationary state. The final value of the kinetic energy is $E_{k i n}=9.0279 \times$ $10^{-5}$. Since the oscillatory energy (3.17) can be bounded by the kinetic energy we can also conclude that very little hydrostatic imbalance has been generated in the course of the simulation.

\section{Conclusions}

The concepts of 'slow' manifolds, balance, and fast oscillations underpin a significant number of flow regimes in atmospheric and ocean dynamics [1] even though a clear separation of time-scales between different flow regimes does not apply in general. Similar to MD, the importance of appropriate numerical methods should not be underestimated. In our view, classical particle mechanics, adiabatic invariance, symplectic and large time-stepping (LTS) methods have a lot to offer in that respect. The development and testing of 
the Hamiltonian particle-mesh (HPM) methods, as proposed in this paper, for realistic three-dimensional problems is clearly the next challenge to tackle.

Acknowledgements. Partial financial support by EPSRC Grant GR/R09565/01 is gratefully acknowledged.

\section{Appendix}

We show how to apply the normal form result of NeISHTADT [22] to the slowfast system (2.4)-(2.5) and essentially follow the exposition of [28]. We first have to introduce conjugate momenta $p_{y}=\dot{y}$ and $p_{x}=\dot{x}$ and restate (2.4)-(2.5) in the form:

$$
\begin{array}{ll}
\dot{x}=p_{x}, & \dot{p}_{x}=-\varepsilon^{-2} y^{2} \nabla_{x} K(x) / 2-\nabla_{x} V(x, y), \\
\dot{y}=p_{y}, & \dot{p}_{y}=-\varepsilon^{-2} K(x) y-\nabla_{y} V(x, y) .
\end{array}
$$

These equations are canonical with Hamiltonian function

$$
\mathcal{H}=\frac{1}{2}\left(p_{x}^{2}+p_{y}^{2}+\varepsilon^{-2} K(x) y^{2}\right)+V(x, y) .
$$

We next perform a non-canonical change of variables $\tilde{y}=y / \varepsilon$ and also rescale time $t$ to $\tau=t / \varepsilon$. This results in the non-canonical equations

$$
\begin{aligned}
x^{\prime}=\varepsilon p_{x}, & p_{x}^{\prime}=-\varepsilon \tilde{y}^{2} \nabla_{x} K(x) / 2-\varepsilon \nabla_{x} V(x, \varepsilon \tilde{y}), \\
\tilde{y}^{\prime}=p_{y}, & p_{y}^{\prime}=-K(x) \tilde{y}-\nabla_{\tilde{y}} V(x, \varepsilon \tilde{y}),
\end{aligned}
$$

with new Hamiltonian

$$
\mathcal{H}=\frac{1}{2}\left(p_{y}^{2}+K(x) \tilde{y}^{2}\right)+\frac{1}{2} p_{x}^{2}+V(x, \varepsilon \tilde{y})
$$

and non-canonical symplectic structure

$$
\Omega=\varepsilon d p_{x} \wedge d x+d p_{y} \wedge d \tilde{y} .
$$

We further transform $\left(\tilde{y}, p_{y}, x, p_{x}\right)$ to new variables $\left(\hat{y}, \hat{p}_{y}, \hat{x}, \hat{p}_{x}\right)$ using a generating function [2]

$$
F=\varepsilon^{-1} \hat{x} p_{x}+\hat{y} K(\hat{x})^{-1 / 4} p_{y}
$$

and the symplectic structure $\Omega$. This yields

$$
x=\hat{x}, \quad \tilde{y}=K(\hat{x})^{-1 / 4} \hat{y}, \quad \hat{p}_{y}=K(\hat{x})^{-1 / 4} p_{y}, \quad \hat{p}_{x}=p_{x}+\varepsilon \hat{y} p_{y} \nabla_{x} K(\tilde{x})^{-1 / 4} .
$$

In the new coordinates the Hamiltonian $\mathcal{H}$ is equivalent to

$$
\begin{aligned}
\mathcal{H}=\frac{\omega(\hat{x})}{2} & \left(\hat{p}_{y}^{2}+\hat{y}^{2}\right)+\frac{1}{2} \hat{p}_{x}^{2}+V(\hat{x}, 0)+ \\
\varepsilon & {\left[K(\hat{x})^{-1 / 4} \hat{y} \nabla_{y} V(x, 0)-\hat{y} \hat{p}_{y} \hat{p}_{x} K(\hat{x})^{1 / 4} \nabla_{x} K(\hat{x})^{-1 / 4}\right]+\mathcal{O}\left(\varepsilon^{2}\right), }
\end{aligned}
$$


where $\omega=K^{1 / 2}$. We finally transform to action-angle variables $(J, \phi)$ via

$$
\hat{y}=\sqrt{J} \cos \phi, \quad \hat{p}_{y}=\sqrt{J} \sin \phi,
$$

and average over the rapidly rotating angle $\phi[2]$. This yields the averaged Hamiltonian

$$
\mathcal{H}_{\text {reduced }}=\frac{\omega(\hat{x})}{2} J+\frac{1}{2} \hat{p}_{x}^{2}+V(\hat{x}, 0)+\mathcal{O}\left(\varepsilon^{2}\right),
$$

and the reduced equation (2.8) is obtained up to perturbations of order $\mathcal{O}(\varepsilon)$. It has been the crucial observation of NEISHTADT [22] that a further transformation of variables $\varepsilon$-close to the identity can remove the undesirable order $\varepsilon$ term up to a remainder term exponentially small in $\varepsilon$. Hence the corresponding action variable, call it $\bar{J}$, is preserved over exponentially long periods of time. We also have $\bar{J}-J=\mathcal{O}(\varepsilon)$ and this explains the order $\varepsilon$ variations in the observable action variable (2.6). Note that these exponential estimates require that both $K(x)$ and $V(x, y)$ are real-analytic functions.

\section{REFERENCES}

1. D.G. Andrews. An Introduction to Atmospheric Physics. Cambridge University Press, Cambridge, 2000.

2. V.I. Arnold. Mathematical Methods of Classical Mechanics. Springer-Verlag, New York, 2nd edition, 1989.

3. G. Benetin and A. Giorgilli. On the Hamiltonian interpolation of near to the identity symplectic mappings with application to symplectic integration algorithms. J. Statist. Phys., 74:1117-1143, 1994.

4. G. Benettin, L. Galgani, and A. Giorgilli. Realization of holonomic constraints and freezing of high frequency degrees of freedom in the light of classical perturbation theory. part II. Comm. Math. Phys., 113:557-601, 1989.

5. F.A. Bornemann. Homogenization in Time of Singularly Perturbed Conservative Mechanical Systems, volume 1687 of Lecture Notes in Mathematics. Springer-Verlag, Berlin Heidelberg, 1998.

6. T.J. Bridges, P. Hydon, and S. Reich. Vorticity and symplecticity in Lagrangian fluid dynamics. Technical report, University of Surrey, 2002.

7. C.J. Cotter. Model Reduction for Shallow-Water Dynamics: Balance, Adiabatic Invariance and Subgrid Modelling. PhD thesis, Imperial College London, 2004.

8. D.R. Durran. Numerical Methods for Wave Equations in Geophysical Fluid Dynamics. Springer-Verlag, Berlin Heidelberg, 1998.

9. J. Frank, G. Gottwald, and S. Reich. The Hamiltonian particle-mesh method. In M. Griebel and M.A. Schweitzer, editors, Meshfree Methods for Partial Differential Equations, Lecture Notes in Computational Science and Engineering, volume 26, pages 131-142, Berlin Heidelberg, 2002. SpringerVerlag. 
10. J. Frank and S. Reich. Conservation properties of smoothed particle hydrodynamics applied to the shallow-water equations. BIT, 43:40-54, 2003.

11. A.M. Gadian, J. Dormand, and J.S.A. Green. Smoothed-particle hydrodynamics as applied to 2D plume convection. Atmospheric Research, 24:289304, 1989.

12. B. García-Archilla, J. M. Sanz-Serna, and R. D. Skeel. The Mollified Impulse Method for Oscillatory Differential Equations. SIAM J. Sci. Comput., 20:930-963, 1998.

13. R.A. Gingold and J.J. Monaghan. Smoothed particle hydrodynamics: theory and application to non-spherical stars. Mon. Not. R. Astr. Soc., 181:375-389, 1977.

14. E. Hairer and Ch. Lubich. The life-span of backward error analysis for numerical integrators. Numer. Math., 76:441-462, 1997.

15. E. Hairer, Ch. Lubich, and G. Wanner. Geometric Numerical Integration. Springer-Verlag, Berlin Heidelberg, 2002.

16. B. Hess, H. Saint-Martin, and H.J.C. Berendsen. Flexible constraints: an adiabatic treatment of quantum degrees of freedom, with application to the flexible and polarizable mobile charge densities in harmonic oscillators model for water. J. Chem. Phys., 116:9602-9610, 2002.

17. J.A. Izaguirre, S. Reich, and R.D. Skeel. Longer time steps for molecular dynamics. J. Chem. Phys., 110:9853-9864, 1999.

18. B. Leimkuhler and S. Reich. A reversible averaging integrator for multiple time-scale dynamics. J. Comput. Phys., 171:95-114, 2001.

19. B. Leimkuhler and R.D. Skeel. Symplectic numerical integrators in constrained Hamiltonian systems. J. Comput. Phys., 112:117-125, 1994.

20. L.B. Lucy. A numerical approach to the testing of the fission hypothesis. Astron. J., 82:1013-1024, 1977.

21. R.S. MacKay. Lectures on slow manifolds. Technical report, University of Warwick, Coventry, 2003.

22. A.I. Neishtadt. The separation of motions in systems with rapidly rotating phase. J. Appl. Math. Mech., 48:133-139, 1984.

23. S. Reich. Smoothed dynamics of highly oscillatory Hamiltonian systems. Physica D, 89:28-42, 1995.

24. S. Reich. Torsion dynamics of molecular systems. Physical Review E, 53:4176-4181, 1996.

25. S. Reich. Backward error analysis for numerical integrators. SIAM J. Numer. Anal., 36:475-491, 1999.

26. S. Reich. Conservation of adiabatic invariants under symplectic discretization. Appl. Numer. Math., 29:45-55, 1999.

27. S. Reich. Multiple times-scales in classical and quantum-classical molecular dynamics. J. Comput. Phys., 151:49-73, 1999. 
28. S. Reich. Smoothed Langevin dynamics of highly oscillatory systems. Physica $D, 138: 210-224,2000$.

29. H. Rubin and P. Ungar. Motion under a strong constraining force. Comm. Pure and Appl. Math., 10:65-87, 1957.

30. R. Salmon. Practical use of Hamilton's principle. J. Fluid Mech., 132:431444, 1983.

31. M. Shimada and H. Yoshida. Long-term conservation of adiabatic invariants by using symplectic integrators. Publ. Astron. Soc. Japan, 48:147-155, 1996.

32. R.D. Skeel. Integration schemes for molecular dynamics and related applications. In M. Ainsworth, J. Levesley, and M. Marletta, editors, The Graduate Student's Guide to Numerical Analysis, pages 119-176, Berlin Heidelberg, 1999. Springer-Verlag.

33. F. Takens. Motion under the influence of a strong constraining force. In Z. Nitecki and C. Robinson, editors, Lecture Notes in Mathematics, volume 819, pages 425-445, Berlin, 1980. Springer.

34. J. Zhou, S. Reich, and B.R. Brooks. Elastic molecular dynamics with flexible constraints. J. Chem. Phys., 112:7919-7929, 2000. 